\title{
Design of Digital Aerial Photography System for UAV based on Wireless Sensor
}

\author{
Network \\ Professor Dr. Sorush Niknamian \\ BSC in Applied Mathematics (IAUCTB), BSC in Mechatronics Engineering (SUT) \\ and PhD in Cell and Molecular Biology and Military Medicine, Liberty University \\ (LU), USA \\ Email: so.ninamian@gmail.com
}

\begin{abstract}
The traditional UAV aerial photography system has the disadvantages of unclear imaging, low system efficiency and poor flight control effect. Thus, a digital aerial photography system based on wireless sensor network is proposed. Firstly, the principle of aerial photography system is analyzed, and the wireless sensor network is set up. A large number of wireless sensor nodes are deployed in the interval, and functions such as wireless communication and calculation are completed by nodes; the SN-RN data acquisition layer, the RN-UAV relay transmission layer and the UAV-DC mobile aggregation layer are designed to form a wireless sensor network architecture, and the UAV digital aerial photography technology is combined to form the wireless sensor network. Experiments show that the medium error, maximum error and medium error limit of the digital aerial photography of the UAV are low, the total working time of the system is short, and the accuracy of the flight execution of the UAV is maintained between 93\%-95\%, and always stable. Therefore, the overall imaging effect of this method is better, the system work efficiency is higher, the system control effect is better,
\end{abstract}


and it is more practical and advantageous.

Keywords: wireless sensor network; UAV; node; aerial photography; system design;

\section{Introduction}

With the development of science and technology, UAV technology has been continuously improved. The digital aerial photography of UAV plays an increasingly important role in various fields due to its simple operation and high efficiency [1]. A set of UAV digital aerial photography system is mainly composed of flight platform, aerial photography system, ground control system and influence processing system. It uses UAV as flight platform and uses high-resolution CCD digital camera, air and ground control system and other equipment to achieve route planning and monitoring, video capture, acquisition, transmission pre-processing functions such as digital aerial photography system [2].

Wireless sensor network (WSN) is a combination of embedded technology, network technology and sensor technology. It is used in military, industrial, agricultural and other fields for information acquisition and object tracking, which is a hot topic at home and abroad. [3].

In the WSN of the UAV digital aerial photography system, a large number of nodes with low power consumption are often randomly scattered $[4,5]$. A node is a microembedded system that senses and collects information from the environment. The UAV digital aerial photography system WSN requires self-assembled network and data transmission as a whole. Each node is required to be able to collect, process and transmit 
local information and data. It is also required to perform operations such as storage, calculation, and aggregation on data forwarded by other nodes. Since the nodes are generally powered by batteries, the functional design requires that the nodes must be low-power, so the processing power, storage capacity and communication capabilities of the nodes are relatively weak [6]. Due to the complex environmental conditions of aerial photography, it is unrealistic to replace the energy of a node or a supplementary node. Therefore, how to use fewer nodes to achieve efficient and reliable aerial photography has become one of the hotspots in recent years.

Literature [7] proposed a method for designing wireless sensor networks on UAV. This method gave the overall architecture and hardware and software design of the wireless sensor network combined with UAV application by analyzing the demand monitoring status of the UAV. However, the resulting aerial image resolution is not ideal.The method of literature [8] was based on the aerial photography system of the microautopilot. The method was mainly composed of the following parts: micro-automatic pilot aircraft (platform), aerial photography subsystem (i.e. ordinary or digital camera and electronic shutter control), aerial photography subsystem (digital CCD camera or digital video camera) and image real-time transmission, display and the recording subsystem, which could simultaneously carry the meteorological sounding subsystem. Atmospheric temperature, pressure and humidity could be obtained simultaneously during aerial photography or aerial photography, but the system performed poorly on planned routes. Literature [9] proposed the design of a multi-rotor UAV route planning system, introduced the architecture of the multi-rotor UAV aerial photogrammetry 
system, analyzed the characteristics of the multi-rotor UAV, and summarized its advantages. Based on the calculation method of related parameters, a route planning algorithm was developed based on this algorithm, and a multi-rotor UAV route planning system that could be used with the multi-rotor UAV ground station was designed and implemented. The overall work efficiency was not ideal.

Aiming at the shortcomings of traditional methods, this paper proposes a digital aerial photography system based on wireless sensor network. Firstly, the principle of aerial photography system is analyzed. On this basis, the wireless sensor network is set up, a large number of wireless sensor nodes are deployed in the interval, and the wireless communication and computing functions are completed by the nodes. the SN-RN data acquisition layer, the RN-UAV relay transmission layer and the UAV-DC mobile aggregation layer are designed to form a wireless sensor network architecture, and the UAV digital aerial photography technology is combined to form a UAV digital aerial photography system based on the wireless sensor network. At the same time, the system architecture can extend the life of the sensor node by alleviating the transmission task of the sensor node in the WSN; by increasing the function and power supply of the relay node, the resource consumption caused by the failure of the transmission node is reduced; the data acquisition efficiency and system life of the UAV digital aerial photography system are improved by the good network communication quality provided by mobile nodes.

\section{Material and methods}

\subsection{Principle analysis of digital aerial photography system for UAV based on}




\section{wireless sensor network}

There are many characteristics analysis based on IEEE 802.15.4 low-power network and cellular network. Therefore, this study mainly analyzes the characteristics and performance between UAV and RN. The schematic diagram of the data exchange between UAV and RN is shown in Figure 1 [10].

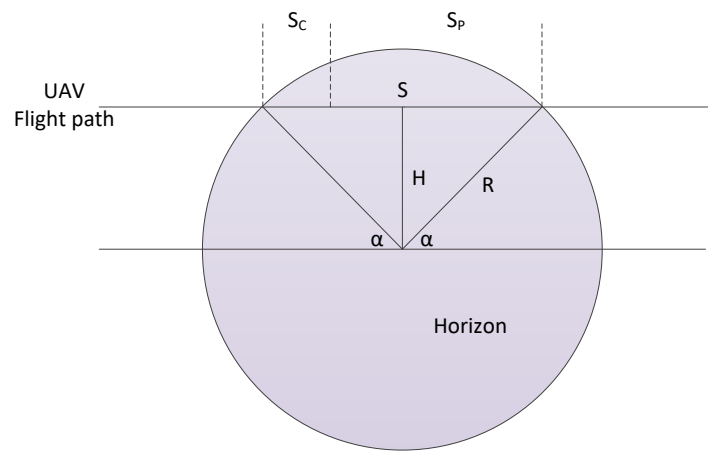

Figure 1. Plan of data exchange between UAV and RN

Note: $\mathrm{H}$ is flight height, $\mathrm{m} ; \mathrm{S}$ is flight distance, $\mathrm{m} ; \mathrm{S}_{\mathrm{c}}$ is communication flight distance, $\mathrm{m} ; \mathrm{S}_{\mathrm{p}}$ is change flight distance, $\mathrm{m} ; \mathrm{R}$ is communication radius, $\mathrm{m} ; \alpha$ is angel, $\left({ }^{\circ}\right)$.

According to Figure 1, the following equation can be obtained: $\mathrm{H}=\mathrm{R} \cdot \sin \alpha$

Where: $\mathrm{H}$ is the height of the UAV from the ground, that is, the flying height; $\mathrm{R}$ is the communication radius of the $\mathrm{RN} ; \alpha$ is the angle between the connecting line between the UAV and the RN and the horizontal plane. $\mathrm{S}=2 \mathrm{R} \cdot \cos \alpha$

Where: $\mathrm{S}$ is the distance that the UAV and $\mathrm{RN}$ actually fly within the communicable range. $\mathrm{S}=\mathrm{S}_{\mathrm{c}}+\mathrm{S}_{\mathrm{p}}$ 
Where: $S_{c}$ is the distance the UAV can fly from the time the UAV enters the RN communication range to the time it can establish communication with each other. $\mathrm{S}_{\mathrm{p}}$ is the distance the UAV flies during the data exchange between the UAV and the RN [11].

$$
\mathrm{R}=\sqrt{\mathrm{H}^{2}+(\mathrm{S} / 2)^{2}}
$$

The time between UAV and RN for data exchange should meet the following equation: $t_{e}=\frac{S_{P}}{V}$

Where: $t_{e}$ is the time required for the data exchange and processing of the UAV and the $\mathrm{RN}$, and $V$ is the flight speed of the UAV. The data exchange between the RN and the UAV should satisfy the following equation [12]:

$$
\mathrm{L}=\left(\mathrm{b} \cdot \mathrm{t}_{\mathrm{e}}\right) / 8
$$

Where: $L$ is the length of the number of data bytes exchanged between the $\mathrm{RN}$ and the $\mathrm{UAV} ; b$ is the data transmission rate between the RN and the UAV.

Equation (5) is substitute into equation (6), it can get:

$$
L=\left(b \cdot \frac{D_{P}}{S}\right) / 8
$$

To ensure that all data cached in the RN can be collected by the UAV during the communication time, the RN's cache size should be greater than or equal to $L$. The actual communication time between the UAV and the RN is greater than or equal to the sum of the time between the establishment of the connection and the time of data exchange $[13,14]$.

$$
t \geq t_{c}+t_{e}
$$

Where: $t$ is the time from the UAV entering the communication range of the $\mathrm{RN}$ to the 
communication range from the $\mathrm{R} ; t_{c}$ is the time required for the UAV to enter the RN communication range to establish a process that can communicate with each other. The UAV speed is variable. When the UAV searches for a relay node along a set trajectory or in a dynamic random flight mode, the UAV cruises at a higher speed (usually 5 to 15 $\mathrm{m} / \mathrm{s}$ ) when the UAV enters the relay node communication range of the ground. In order to ensure good transmission quality and ensure that the data stored in the relay node can be completely transmitted to the UAV, the UAV flies at a lower speed and hovers in the air (usually $0-5 \mathrm{~m}$ ) /s), to perform data collection tasks. After the data transmission is completed, the UAV returns to the original cruise speed, continues to search for the next relay node, or returns to the base [15-17].

2.2 Design of digital aerial photography system for UAV based on wireless sensor network

\subsubsection{Composition of wireless sensor networks}

The wireless sensor network is composed of SN (sensor node), relay node (RN, relay node), unmanned aerial vehicle (UAV), data center (DC, data center) and other elements, as shown in Figure 2.

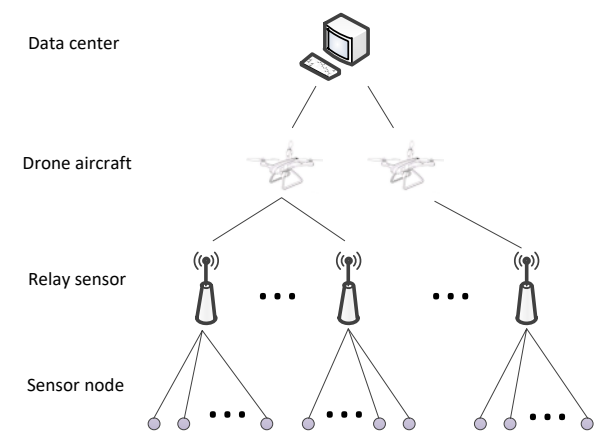

Figure 2. Wireless sensor network system

The nodes of the wireless sensor network have the characteristics of small size, low 
energy consumption, low price, and have the functions of sensing the external environment, performing wireless communication and computing. Such nodes can sense and collect data and information from a large number of environments. A large number of such wireless sensor nodes are deployed in a large geographic area that will form a dense wireless ad-hoc sensing network [18].

The most important function of the relay node is to connect the nodes of the wireless sensor network, the UAV communication, and the data transmission bridges and hubs. The relay node itself does not need to perceive the environmental information, and it acts as the data concentration node of the wireless sensor network. The sensor node in the area where it is located is notified to collect data, the data is stored in the buffer of the relay node, and the data is transmitted to the UAV within a given communication time. The distance between each relay node can be very long and does not need to communicate with each other [19]. In general, the relay node has a relatively strong power supply and a long life period relative to the sensor node, and can provide a large communication range by means of antenna gain and others.

The UAV is a fixed-wing or rotary-wing unmanned aircraft with its own power, controllable and mobile communication equipment. It can be reused without a cockpit, but equipped with autopilot and flight attitude control. The mobile communication device carried by the UAV supports a variety of communication protocols and a variety of communication interfaces, and can communicate with the ground or air devices in a variety of communication modes. Since the UAV can provide power, the portable device has certain computing power and storage function, which can realize data 
compression and processing. When the communication quality is not good, the data received from the ground can be stored in the cache and recovered well. Data is transmitted to the data center when the communication quality is good [20].

The data center is composed of resources such as servers, storage devices, databases, geographic information systems, and high-speed network bandwidth. The data center is responsible for receiving data transmitted by the UAV. In order to ensure the integrity and reliability of the data, the data center will perform statistical analysis and data mining on the collected information, and make decisions based on the results of the analysis or take corresponding actions (such as issuing instructions to the UAV, adjusting the path to collect data, etc.). Data in the data center can be accessed by users, computers or other devices, providing access to the service terminals via the Internet or mobile internet, and displaying the analyzed data information in a friendly and intuitive interface [21].

\subsubsection{Architecture of Wireless Sensor Network}

The architecture of wireless sensor network consists of the SN-RN data acquisition layer, the RN-UAV relay transport layer and the UAV-DC mobile aggregation layer, as shown in Figure 3. 


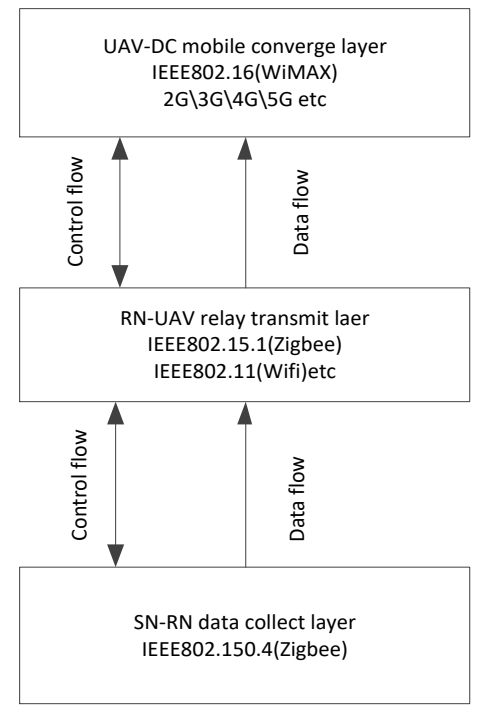

Figure 3. Wireless Sensor Network Architecture

1. SN-RN data acquisition layer

The data acquisition layer consists of a network established between the sensor node and the relay node. The data acquisition layer network has the following characteristics [22]:

(1) Limited hardware resources: therefore, the protocol layer of the operating system of the wireless sensor node needs to be simplified.

(2) Limited energy: since the wireless sensor node has less energy resources and cannot replenish energy at any time, once the energy is exhausted, the wireless sensor node will not continue to work normally.

(3) Distributed: multi-distributed structure is adopted, in which wireless sensor nodes can enter and leave the network at any time.

(4) Self-organization: through layered protocols and distributed algorithms, wireless sensor nodes can spontaneously schedule their work processes appropriately. When deployed to a specific area and activated, the corresponding network can be organized 
spontaneously.

(5) Dynamic topology: the system is a dynamic network, in which the wireless sensor nodes will exit the network due to running out of power or damage, and will be added to the current network because of the task, so the topology of the network is dynamically changed.

(6) Dense distribution: in order to enhance the task monitoring in the target information collection area, a large number of wireless sensor nodes will be deployed to this area. Due to these characteristics of the data acquisition layer, this layer usually uses the IEEE 802.15.4 network communication protocol to achieve the above functions. A typical use of the IEEE 802.15.4 protocol is Zigbee. The transmission rate of the IEEE 802.15.4 protocol is generally less than 1 Mbps. IEEE 802.15.3 can also be used if the data transfer rate is required to increase. But high transmission rates also mean higher energy consumption. This layer is usually meshed by mesh, and the network hierarchy is usually a star structure or a tree hierarchy.

At the SN-RN layer, sensor nodes are dispersedly deployed in the aerial image area. When a star network hierarchy is adopted, each sensor node is centered on only one relay node, and the sensor nodes are only fixed with one at each monitoring cycle. The relay node communicates and monitors the data transmission. The sensor node and the sensor node are kept at a certain distance and do not communicate with each other. The data collected by all sensor nodes are directly aggregated to the designated relay node [23]; When adopting a tree-like hierarchical structure, the sensor nodes at the outermost periphery are only responsible for the function of data collection and uploading data, 
the sensor nodes in the middle are responsible for data collection and data forwarding, and the sensor nodes communicate with each other. The data collected by all sensor nodes is directly or indirectly aggregated to the designated relay node, in order to minimize the energy consumed by the sensor nodes in the data transmission process, the level of the tree hierarchical structure cannot exceed 3 floors. In contrast, the star structure system has a longer life cycle, and the tree-level structure covers a larger monitoring area. Each relay node forms an autonomous sensor network area with a number of sensor nodes surrounding it [24].

2. RN-UAV relay transport layer

The relay transport layer consists of a network established between the relay node and the UAV mobile node. The relay transport layer network has the following characteristics:

(1) Simple structure: direct communication between UAV and RN, that is, only one hop direct connection.

(2) More energy: $\mathrm{RN}$ is generally powered by solar energy, and UAV can be powered by a rechargeable battery.

(3) Stable and reliable: UAV communicates with the ground $\mathrm{RN}$ in the air, which may be less likely to be blocked by plants or houses, and the packet loss rate will be reduced. (4) Long communication distance: good communication quality, can provide antenna coverage with larger power.

From the perspective of compatibility, the relay transmission layer can use the IEEE 802.15.4 protocol for enhanced signals, or the IEEE 802.11 protocol with longer 
transmission distance. The network topology between the RNs of this layer is dominated by a linear structure or a grid structure. This topology facilitates the UAV to perform tasks in a straight line. For UAVs with single or multiple rotors, the UAV can enter the next straight line of data acquisition at the corner of the acquisition information area at a right angle.

In the RN-UAV layer, the relay nodes are deployed in a linear manner according to the rules. Many relay nodes are arranged in a row, and the adjacent two nodes are distributed in a distance of several hundred meters. There is no limit on the number of relay nodes in each row, and no communication and data transmission between any two relay nodes. The relay node is not responsible for the task of direct data collection. It is responsible for storing the monitoring data transmitted by the adjacent sensor nodes in its own buffer, waiting for the UAV to collect data.

\section{UAV-DC mobile aggregation layer}

The mobile aggregation layer consists of a network established between a single or multiple UAVs and a data center. A cellular data network is directly connected between the UAV and the DC. The mobile aggregation layer network has a very long communication distance, has error detection and packet loss retransmission function, supports real-time or delayed data transmission, and has a simple structure.

\subsection{UAV aerial photography system based on wireless sensor network}

\subsubsection{Composition of UAV digital aerial photography system}




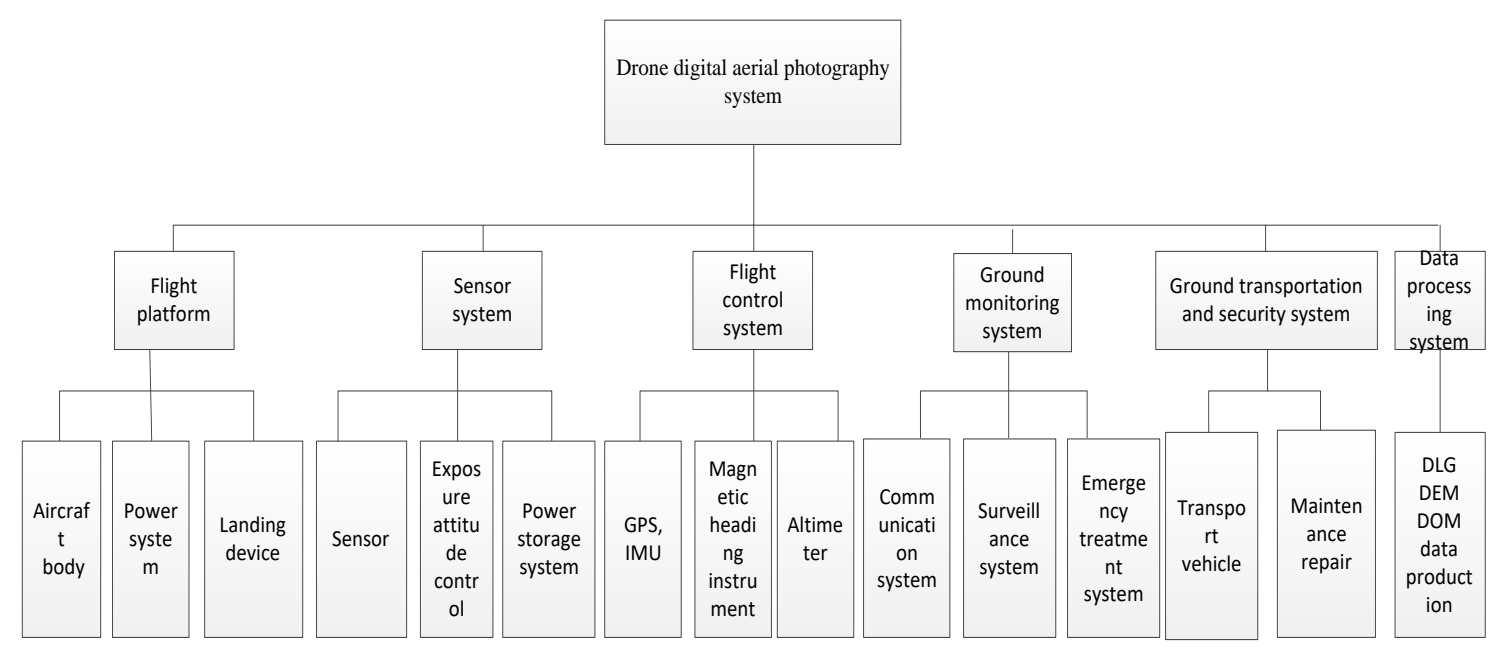

Figure 4. Composition of UAV low-altitude remote sensing aerial photography system

\subsubsection{Technical Process}

Like traditional aerial photography, digital aerial photography of UAVs based on wireless sensor networks also requires steps such as route design, aerial photography, quality inspection, fly-fill or fly-back, and image-controlled measurement. The difference is that the route design of the UAV aerial photography is small, so there is no need to consider the change of the curvature of the earth; the inspection of the aerial quality can be completed at the aerial scene without the need to print photos; Photographic measurement work under certain conditions must first create a full-area fast mosaic. 


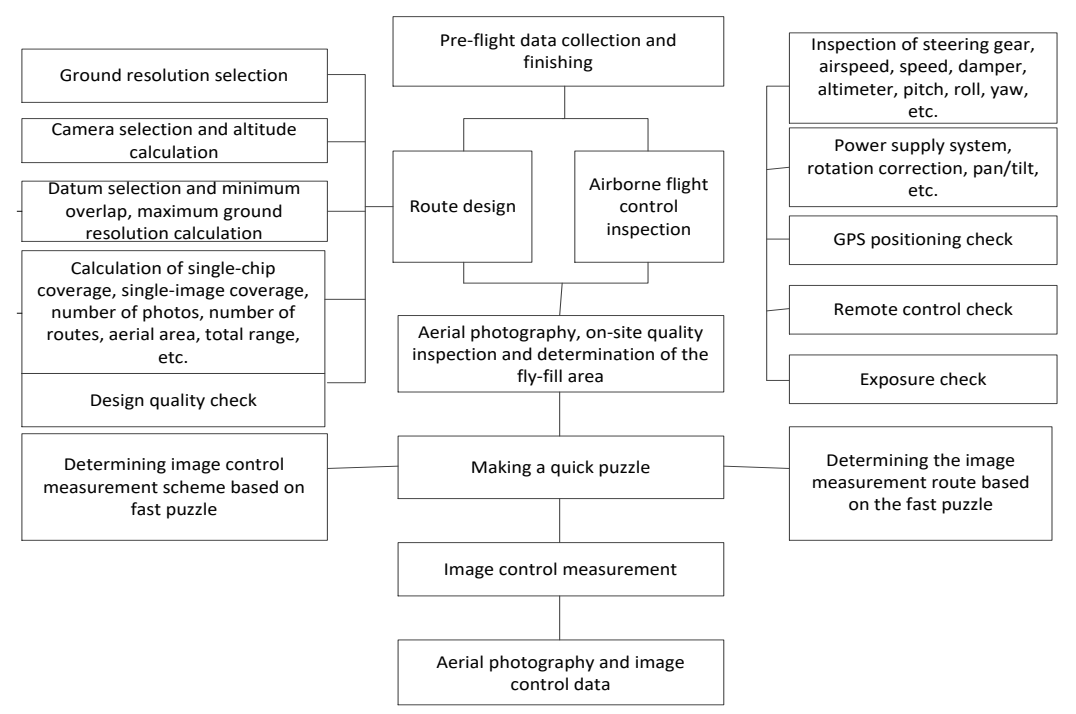

Figure 5. UAV aerial photography system technical process

1. Work permit and airspace coordination

At the first time after entering the photographic area, it should contact the local people's government or the competent department of surveying and mapping, timely inform the relevant projects, and coordinate the local personnel in traffic, accommodation, flight safety, guidance, medical treatment and other aspects to help, etc. If there is a confidential unit, military area or airport near the survey area, it is necessary to go to the air reference center under the jurisdiction of the survey area to handle airspace coordination procedures.

2. On-site survey

After coordinating local conditions and airspace, personnel should be organized to conduct on-site reconnaissance and collect relevant information. These materials include:

(1) The weather conditions in the photography area in recent days.

(2) Using the handheld GPS, the corners of the photography area is drilled and corresponding records are made. Google Earth can be used to verify the correctness of 
the four corners coordinate point of the survey area and confirm whether the photography area is correct.

(3) Looking around the survey area, it is to see if there is a high mountain, and estimate whether the height of the mountain and height difference have an impact on flight safety, whether there is snow on the mountain, and whether the image affects the quality. (4) A suitable landing site is found according to the type of aircraft.

3. On-site route design

Digital photography of UAVs is generally performed on digital aerial photography in a small area. Unlike traditional aerial photography, there is no need to consider the curvature of the earth in the design of the route, and it is not necessary to know the elevation of the ground point very accurately. Traditional aerial photography has clear operating specifications and procedures. It is necessary to use a 1:10000 or 1:50000 topographic map or use the existing DEM for the design of the reference surface. If it is necessary (when the height difference is greater than $1 / 6$ altitude), photographic partitioning is set. For digital aerial photography of UAVs based on wireless sensor networks, in general, the four-corner coordinates of the camera zone can be known for route design. Photographic partitioning is set under very special conditions [25-31].

(I) According to the scale of the map, the approximate ground resolution is determined. The corresponding ground resolution should be selected before the relative altitude is determined.

(II) Viewing the general situation of the photography area: the coordinates of the photography area or the coordinates of the photography area required by Party A are 
entered into Google Earth in the computer to check whether the basic situation of the photography area is consistent with the mastery of the situation, whether there are an area of mountains, military facilities, airports, etc. that is not conducive to aerial photography.

(III) Selection of the base plane of the photography area: according to the condition of photography area displayed in Google Earth, the highest and lowest elevations of the area are counted, and the datum of photography area is determined according to the maximum and minimum elevation values. The datum is generally the average of the maximum and minimum elevations in the photography area.

(IV) Selection of the appropriate heading and side overlap: the heading and side overlap required for the map are selected. For example, the target area of Longma Hotel in Cangzhou City, Hebei Province is to measure 1:2000 orthophotos, the heading overlap can be set to $65 \%$, and the side overlap is set to $35 \%$.

(V) The heading and side overlap of the highest point, and the ground resolution at the lowest point in the area are calculated.

Minimum ground resolution $=\frac{\text { Datum elevation }- \text { Lowest elevation }=\text { Relative altitude }}{\text { Focal length }} \times$ Single cell size

Maximum overlap in the photo zone $=\frac{\text { Heading overlap }-\frac{\text { Highest elevation }- \text { Datum elevation }}{\text { Relative altitude }}}{1-\frac{\text { Highest elevation }- \text { Datum elevation }}{\text { Relative altitude }}}$

(VI) Adjusting the ground resolution and overlap for recalculation: when the minimum ground resolution of the photography area exceeds the resolution required by the map, or when the highest overlap of the photography area is less than the mapping requirement, the ground resolution must be changed. The relative altitude, the lowest 
point ground resolution, and the highest point overlap are recalculated until the requirements are met.

(VII) Coordinate transformation in route design: WGS84 latitude and longitude coordinates - used in UAV flight control

Plane coordinates must be used in the route design. If Party A has given a certain plane coordinate, it can be directly input into the route design software for calculation. If the four-corner coordinates are selected in Google Earth, it can be converted to WGS84 plane coordinates and then designed. Then it is converted into WGS84 latitude and longitude coordinate input flight control.

(VIII) Calculating the coordinates of each exposure point: according to the set value, the route design software is entered and the coordinates of the exposure points are calculated. Statistics of the number of flights, number of photos, baseline length, side to side distance and total voyage of this aerial photograph are given.

(IX) Route design under special circumstances: When the elevation of the area is greater than $1 / 6$ voyage, in principle, a photographic division should be established. For the aerial photography of UAVs, the elevation is broad. When the elevation of the photography area is large, it is to try to increase the resolution and increase the overlap method to overcome. When this method still does not work, a photography partition must be set up.

\section{Debugging of ground monitoring station}

The ground monitoring station personnel designs the exposure point coordinates on the day before the flight or on the flight site, and introduces the coordinate points of 
exposure point into the ground monitoring software to confirm that there is no error before flying.

\section{Take-off check}

At the same time as the route design, in this time, the airborne flight control, camera, etc. are installed. This series must be checked after installation, or whenever the aircraft falls before entering the flight. There are mainly the following checks:

(1) Yaw check: the gyro zero point is correct, and the right turn is positive. In UAV mode, when the PID value of running channel is not 0 , the rudder deflects the damping yaw change in the corresponding direction.

(2) Altimeter check: if there is a condition to get the air pressure value of the current control box, set the current air pressure value and the current altitude value on the ground station. The height of the aircraft is changed, and the height display value will be changed accordingly.

(3) Speed check: if the aircraft is equipped with a speed sensor, the engine is turned by hand to see if there is a speed display on the ground station, and the speed division setting is corrected.

(4) GPS positioning check: the time from power on to GPS3D positioning should be about 1 minute. If it is more than 5 minutes, it can't be positioned, and the GPS antenna connection or other interference are checked. After positioning, the number of satellites is generally more than 6 and the PDOP horizontal positioning quality data is as small as possible, generally between 1 and 2 .

(5) Vibration test: when the engine starting, the jitter of the sensor data at different 
speeds, and the beating of the control surface are observed, especially the attitude data shown by the attitude table (level meter). All beating must be in a small range, otherwise the damping measures should be improved.

(6) Battery test: the effective working time of the battery is determined through the discharge test to ensure that the future flight is within a reliable and guaranteed power supply time. The alarm voltage of the ground station is set to: main power of $7 \mathrm{~V}$, servo power of $4.6 \mathrm{~V}$.

(7) Impact of digital transmission on the sensor: in UAV mode, if the impact is large, the actual value in the sensor data is checked to see if the gyro value is around zero; otherwise the transmitter antenna position must move. Other transmitters must also be tested this way.

(8) Flight data check: if the flight path data has been uploaded for flight control is checked.

\section{Aerial camera preparation}

The camera first needs to adjust the focus to infinity, fix it with mechanical devices, and purchase a professional camera to place the equipment box for safe transportation. The following preparations should be made before taking off:

(1) Firstly, it is shoot on the ground, the purpose is to detect whether the image is illusory, and the aperture and shutter required for this flight are set.

(2) When loading and unloading from the aircraft, it is handled gently to avoid touching the lens.

(3) It is checked if the M file is used before the day, the lens cover is open, the camera 
is working properly, and there is power.

(4) During the flight, a person is scheduled to record the exposure of each route, in order to compare with the final number of images.

(5) After the flight is over, if the total quantity is correct is seen. The purpose is to stop the camera from working when the flight is halfway, and the total number is correct to download the image. Whether each piece can be opened is checked, a few images (urban, urban edge, mountain area) are selected to view the actual pixels in the PS.

(6) One person is arranged to record the flight situation, and whether there is any problem with the rise and fall, if there is any problem, it should specify the situation, process and result of the problem. In addition, basic information such as weather, site conditions, and shooting conditions should be recorded.

(7) The flight team should send the record (document) back to the technical person in charge every day. If the delay is caused by the lack of access to the Internet, etc., the delay should be notified by SMS.

\section{UAV digital aerial photography}

During the digital aerial photography of the UAV, attention should be paid to the monitoring of the flight state of the aircraft and the working state of the airborne sensor. There should be a response plan for various possible emergencies to ensure the safety of the flight process and the quality of the mission completion.

\section{Field data finishing}

After the UAV has landed, the image data should be downloaded in time, and some necessary basic checks should be performed on the acquired data. 
(I) Image overview check

Data is organized in such a way that each original image can be opened, each storage is complete, and the exposure is not moderate.

(1) It is checked whether the number of exposure points is consistent with the number of images. If it is inconsistent, the cause is found in time.

(2) It is checked whether the recorded value of each route is consistent with the number of images actually flying. For example, if 20 images are recorded in the ground station of the first route and 005 is the first, it is checked whether $05-024$ is the first route, especially the relationship between 024 and 025 .

(3) The images are loaded in folders according to the number of routes, and the number of images on each route should be the same.

(II) The flight record form is filled in.

(III) Image naming rules

At present, the exposure point name of the flight control output starts with 1 and the serial number is recorded. In order to ensure that the coordinate name of the exposure point is consistent with the image name, it is convenient to map the image to the exposure point when the exposure point is displayed, and the image name does not need to be changed. However, before the aerial photography is carried out, the original image in the camera should be deleted and set from 1 to ensure that the aerial photography image starts from 001. After the inspection is completed, the name of the image will be changed according to the requirements of the national basic aerial photography. (IV) Packing and sending 
All the images are packed by route folder, and it also includes the flight record table and the coordinate data of exposure point.

9. On-site quality inspection

(1) Initial image inspection: the basic situation of the image is checked, including the number of images, the number of navigation belts, whether the image quality is clouded, foggy, snowy, whether the image is illusory, and whether the image is named correctly.

(2) It is checked whether the data is complete: to check whether the flight record table, exposure point coordinate data, etc. are existed, and whether the completeness and specification are filled in.

(3). Displaying the coordinates of the exposure point: it is checked whether the coordinates of the exposure point are consistent with the actual flight conditions. When exposure coordinates are clearly deviated from the route, it should make good records and check the images.

(4) Image resampling: image resampling is performed in the PS, the purpose is to reduce the amount of image data, which is convenient for checking operations.

(5) Image rotation: the image is rotated according to the method of filling in the flight record table, paying particular attention to whether the rotation of the odd-numbered and even-numbered navigation belts is the same.

(6) Quality inspection: inspection records are output, and the unqualified images should be recorded accordingly.

(7) Notice to make up and re-fly: if the image quality is found to not meet the design 
requirements, or there are relative loopholes or even absolute loopholes, it should be timely replenished or even re-flyed.

In addition, there are a few cases where the angle of the roll of the UAV is large, and the displacement of the adjacent headings between the heading and the sideways appears. When the elevation difference is large, some image pairs with small threedimensional overlap will affect the turning point of the relative directional connection point in the later period, forming the so-called image island, which affects the construction of the free network, and must be supplemented.

When flying a certain route, it should not be re-float according to the original design. When the roll angle is large and the fly is made up, it is recommended that the first exposure of the fly-fill route be designed and flowed forward by adding half of the photographic baseline at the original first exposure point to ensure the heading and the adjacent airfoil. Correspondingly, it is also beneficial to insert the supplementary flying aerial film in a certain area with a small degree of overlap, and the baseline between the inserted aerial film and the original flying aerial film is too short, which causes the intersection angle to be too small, and affects the construction of the free network; When the overlap is small and the fly is compensated, the reference plane of the area can be appropriately raised to increase the overlap on the premise that the ground resolution does not change greatly. After the calculation, the fly-fill reference plane is determined and the flight is carried out.

\section{Image processing}

The image that has passed the inspection must be pre-processed before calling the 
customer or the next process, including:

(I) Format Transferring: different internal storage formats of camera may be different.

Generally, all images need to be saved as TIF format.

(II) Distortion error correction: correction of the distortion is performed by the preprocessing software according to the identified camera calibration parameters. The blackened edge of the corrected image indicates that the correction has been completed.

When the parameters are input in the post-empty three measurements, all the distortion parameters are zero.

(III) Submission of image results: the image results are organized, generally including the following:

(1) original image of the submarine zone; (2) image after correction of the distortion of the submarine; (3) flight record table; (4) coordinate file of exposure point; (5) image inspection record; (6) data compilation record; (7) camera parameter file; (8) main point offset file after distortion correction.

\section{Results}

In order to test the validity of the paper, the design experiment is verified.

\subsection{Experiment setting}

Flight platform setting: the flight platform is a hand-dive UAV, the production material is Elapor foam, and the length of wingspan is $163 \mathrm{~cm}$.

The length of plane is $120 \mathrm{~cm}$, the take-off weight is $2.7 \mathrm{~kg}$ and it has a lithium polymer battery $(18.5 \mathrm{~V}, 5300 \mathrm{mAh})$. The suitable flying height is $59-750 \mathrm{~m}$, the average wind speed of wind resistance is $50 \mathrm{~km} / \mathrm{h}$, the suitable temperature is $-20^{\circ} \mathrm{C}-45^{\circ} \mathrm{C}$, the 
maximum endurance time is $50 \mathrm{~min}$, and the cruising speed is $65 \mathrm{~km} / \mathrm{h}$. The area covered by a single flight is shown in Table 1 .

Table 1. Single flight coverage area of UAV system

\begin{tabular}{|l|l|l|ll|}
\hline $\begin{array}{l}\text { Ground } \\
\text { resolution/cm }\end{array}$ & $\begin{array}{l}\text { Row } \\
\text { height } / \mathrm{m}\end{array}$ & $\begin{array}{l}\text { Side overlap 65\% } \\
\text { Side overlap } 85 \% \\
\text { Aerial area } / \mathrm{km}^{2}\end{array}$ & $\begin{array}{l}\text { Side overlap 20\% } \\
\text { Side overlap } 80 \% \\
\text { Aerial area } / \mathrm{km}^{2}\end{array}$ \\
\hline 1.6 & 59.4 & 0.7 & 1.62 & \\
\hline 2.6 & 96.5 & 1.13 & 2.65 \\
\hline 3.2 & 119 & 1.42 & 3.20 \\
\hline 5.0 & 186 & 2.25 & 5.12 & \\
\hline 10.0 & 371 & 4.54 & 9.97 & \\
\hline 20.0 & 743 & 8.67 & 18.20 \\
\hline
\end{tabular}

Since the stability of the UAV's flight is easily affected by the airflow, in order to ensure that the aerial photography image does not have a loophole, the heading and the side overlap should be increased. Therefore, the design heading overlap is $70 \%$, and the design side overlap is $50 \%$.

\subsection{Performance testing}

(1) Aerial imaging effect

By using the method of this paper, the method of literature [7] and the method of literature [8], low-altitude aerial photography (70 frames in 1:2 000 frames) is carried out in a region of about $50 \mathrm{~km}^{2}$, and the error in relative orientation is $4 \mu \mathrm{m}$ (the pixel size is 6.4 micron $\mathrm{m})$. The error plane in the encryption point is $0.5 \mathrm{~m}$ and the height is $0.5 \mathrm{~m}$. The median error of DOM detection point is $0.63 \mathrm{~m}$. The accuracy of aerial imaging results under different methods is shown in Table 2.

Table 2 Accuracy comparison of aerial imaging results

\begin{tabular}{|l|l|l|l|l|}
\hline Method & $\begin{array}{l}\text { Number of DOM } \\
\text { checkpoints }\end{array}$ & $\begin{array}{l}\text { Medium } \\
\text { error/m }\end{array}$ & $\begin{array}{l}\text { Maximum } \\
\text { error/m }\end{array}$ & $\begin{array}{l}\text { Medium error } \\
\text { tolerance } / \mathrm{m}\end{array}$ \\
\hline The proposed method & 30 & 0.63 & 1.07 & 1.2 \\
\hline Method of literature [7] & 30 & 0.78 & 1.43 & 1.44 \\
\hline Method of literature [8] & 30 & 0.92 & 1.32 & 1.48 \\
\hline
\end{tabular}

Analysis of Table 2 shows that under the same number of detection points, the medium 
error, maximum error, and medium error tolerance of the digital aerial photography of the UAV by using the proposed method are $0.63 \mathrm{~m}, 1.07 \mathrm{~m}$, and $1.2 \mathrm{~m}$, respectively, which are lower than the that of the method of literature [7] and the method of literature [8]. Therefore, the aerial imaging accuracy of the proposed method is higher and the imaging effect is better.

\section{(2) Comparison of system work efficiency}

The experimental is set to test the work efficiency of system by using the proposed method, the method of literature [7] and the method of literature [8] method, a total of 4 sets of experiments are designed. The working time of the system is recorded as shown in Table 3.

Table 3. Comparison of system working hours under different methods

\begin{tabular}{|l|l|l|l|l|}
\hline $\begin{array}{l}\text { Number of } \\
\text { trials }\end{array}$ & $\begin{array}{l}\text { RN-UAV } \\
\text { operation } \\
\text { Adjust /s }\end{array}$ & $\begin{array}{l}\text { Total working time of } \\
\text { using the proposed } \\
\text { method/s }\end{array}$ & $\begin{array}{l}\text { Total working time } \\
\text { of using the } \\
\text { method } \\
\text { literature [7]/s }\end{array}$ & $\begin{array}{l}\text { Total working time of } \\
\text { of } \\
\text { using the method of } \\
\text { literature [8]/s }\end{array}$ \\
\hline 1 & 723.114 & 6215.712 & 32417.991 & 29845.376 \\
\hline 2 & 3892.231 & 78923.115 & 253241.988 & 395412.624 \\
\hline 3 & 4231.892 & 81229.315 & 293134.221 & 264584.335 \\
\hline 4 & 633.201 & 5332.633 & 27214.454 & 9478.560 \\
\hline
\end{tabular}

Analysis of Table 3 shows that when the RN-UAV operation adjustment time is the same, the proposed method is used for aerial photography, the total working time of the system is $6215.712 \mathrm{~s}, 78923.115 \mathrm{~s}, 81229.315 \mathrm{~s}$, and $5332.633 \mathrm{~s}$, respectively, which are lower than the that of the method of literature [7] and the method of literature [8]. Therefore, the aerial photography work of the design method in this paper is short in time and the system works more efficiently.

(3) Comparison of planned route execution levels

The proposed method, the method of literature [7] and the method of literature [8] method for experimental testing is set to plan the route execution effect. According to 
the input aerial range and aerial camera parameters, the optimal route of the aerial camera area is solved, the coordinates of the aerial camera center point are solved, coordinate conversion is performed at the same time, and the flight route data is transmitted to the control center via the wireless sensor network. The accuracy of the flight path of the UAV under the three methods is counted. The result is shown in Fig. 7.

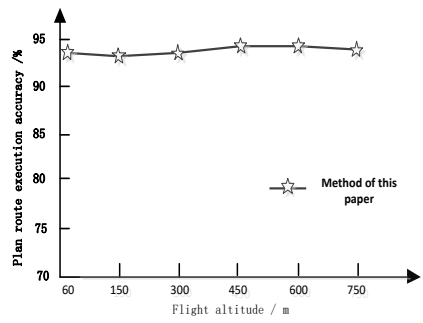

(a)This method plans route execution accuracy

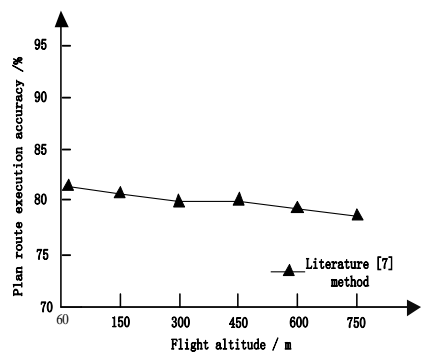

(b)Document [7] method to plan route execution accuracy

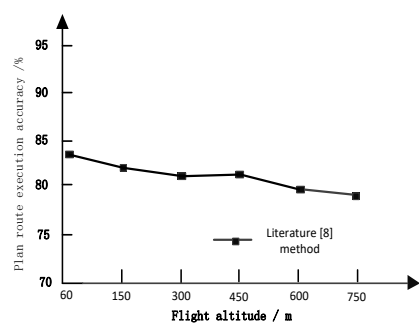

(c)Document [8] method to plan route execution accuracy

Figure 7. Comparison of planned route execution accuracy under different methods Analysis of Figure 7 shows that when the flight height of the UAV is $69-750 \mathrm{~m}$, the accuracy of the UAV execution of the planned route flight by using the method of 
literature [7] is between $81 \%$ and $78 \%$, and that of the method of literature [8] is maintained between $84 \%$ and $79 \%$, and both of them show a trend of decreasing accuracy; while the accuracy of the proposed method is maintained at $93 \%$. - 95\%, and always stable. Therefore, the proposed method used for executing the UAV's planned flight route has high accuracy and better system control effect.

\section{Discussion}

(1) Comparison of aerial imaging effect

According to the above experimental results, under the same number of detection points, the medium error, maximum error, and medium error tolerance of the digital aerial photography of the UAV by using the proposed method are $0.63 \mathrm{~m}, 1.07 \mathrm{~m}$, and $1.2 \mathrm{~m}$, respectively; medium error, maximum error, and medium error tolerance of the digital aerial photography of the UAV by using the method of literature [7] are $0.78 \mathrm{~m}$, $1.43 \mathrm{~m}$ and $1.44 \mathrm{~m}$ respectively, and those of the method of literature [8] are $0.92 \mathrm{~m}$, $1.32 \mathrm{~m}$, and $1.48 \mathrm{~m}$, respectively. Therefore, the error values under the proposed method are lower than those in the literature [7] and the literature [8]. It proves that the aerial imaging of the proposed design method has higher precision and better imaging effect.

(2) Comparison of system work efficiency

According to the above experimental results, when the RN-UAV operation adjustment time is the same, the proposed method is used for aerial photography. The total working time of the system is $6215.712 \mathrm{~s}, 78923.115 \mathrm{~s}, 81229.315 \mathrm{~s}$, and $5332.633 \mathrm{~s}$, respectively. When the method of literature [7] is used for aerial photography, the total working time of the system is $32417.991 \mathrm{~s}, 253241.988 \mathrm{~s}, 293134.221 \mathrm{~s}$, and 27214.454 
s respectively. When the method of literature [8] is used for aerial photography, the total working hours of the system are $29845.376 \mathrm{~s}, 395412.624 \mathrm{~s}, 264584.335 \mathrm{~s}, 9478.560 \mathrm{~s}$ respectively. Therefore, the system work time under the proposed method is lower than the system work time of the method in literature [7] and literature [8]. It proves that the aerial photography work of the design method in this paper is short in time and the system works more efficiently.

(3) Comparison of execution levels of planned route

According to the above experimental results, when the flying height of the UAV is 69$750 \mathrm{~m}$, the accuracy of the UAV's execution of the planned route flight under the proposed method is maintained between $93 \%$ and $95 \%$, and it is always stable; The accuracy of the UAV's execution of the planned route flight under the method of literature [7] is between $81 \%$ and $78 \%$; while that of the method of literature [8] is maintained between $84 \%$ and $79 \%$, showing a downward trend. It is proved that the UAV execution of planed route of the proposed method has high accuracy and the system control effect is better.

In summary, according to the experimental results, the digital aerial photography system based on wireless sensor network designed in this paper has good accuracy of aerial imaging results, short aerial working time, and high accuracy of planned route execution. The imaging effect is better, the system works more efficiently, the system control effect is better, and it is more practical and advantageous.

\section{Conclusions}

Aiming at the problems of unclear imaging, low system efficiency and poor control 
effect in traditional methods, a digital aerial photography system based on wireless sensor network is proposed. Based on the principle of aerial photography system, the wireless sensor network is set up, a large number of wireless sensor nodes are deployed in the interval, and functions such as wireless communication and calculation are completed by the node; SN-RN data acquisition layer, RN-UAV relay transmission layer and UAV-DC mobile aggregation layer are designed to compose a wireless sensor network architecture. Combined with the digital aerial photography technology of UAV, the digital aerial photography system of UAV based on the wireless sensor network is composed. Experiments show that the overall imaging effect of the proposed method is better, the system work efficiency is higher, the system control effect is better, and it is more practical and advantageous.

\section{References}

[1] Jin, D.Y.; Chen, J.X. Application of wireless sensor network design on UAVs. China Science and Technology Information 2017(8):79-80.

[2] Li, W. Research on data collection method based on Uav in wireless sensor network. Northwest University, 2016.

[3] Yao, Z.M.; Jing, B.G.; Sun, C.P. Design and test of plant image monitoring system based on mobile wireless sensor network. Journal of Agricultural Engineering 2016, 32(11):189-196.

[4] Mou, S.; Yin, H.; Su, X. Wireless sensor network technology and application. China Water Conservancy and Hydropower Press 2016.

[5] Liu, Q.K. Application of data fusion technology in wireless sensor networks. 
Technology Outlook 2016, 26(28):14-21.

[6] Zhou, X.M. Application of data fusion technology in wireless sensor network. Digital Space 2018(1):158-158.

[7] Jin, D.Y.; Chen J.X. Application of wireless sensor network design on UAVs. China Science and Technology Information 2017, 8(3):254-261.

[8] Chen, H.B.; Ma, S.Q.; Wang, G., et al. Remote sensing systems on board autonomous miniature aircraft. Remote Sensing Technology and Application 2001, $3(5) 18-23$

[9] Yang, X.C.; Jia, X.F. Design and implementation of multi-rotor uav route planning system. Automation Application 2018, 1(11)45-50.

[10] Pan, Y.; Ma, A.P. Discussion on rapid processing method of aerial photography measurement image data of UAV. Architectural Engineering Technology and Besign 2016(32):71-78.

[11] Zhang, W.U.AV farmland information monitoring system based on wireless sensor network. South Agricultural Machinery 2017, 48(9):36-37.

[12] Wang, F.J.; Duan, H.L.; Guan, X. The production of 1:500 DLG using the UAV aerial system based on complex terrain. Urban Geotechnical Investigation and Surveying 2015(5):86-90.

[13] Cao, F.H.; Song, Z.W.; Li, Y.; et al. Determination of precise spatial position of aerial photography of UAV. Bulletin of Surveying and Mapping 2016(6):144-145.

[14] Ding, L.; Wang, Z.S. Research and implementation of greenhouse monitoring system based on wireless senor network. Computing Technology and Automation 2016, 
35(3):137-140.

[15] Hu, H.F.; Ma, L. Research on security requirements of wireless sensor networks. Practical Electronics 2017(21):83-84.

[16] Zong, J.L. Effective monitoring method for sensitive information of wireless sensor networks. Electronic Technology and Software Engineering 2017(10):14-14.

[17] Guo, Y.; Chen, Y.; Hui, F. Research of laboratory remote monitoring and early warning system based on wireless sensor network. Research and Exploration in Laboratory 2016, 35(3):105-109.

[18] Zhang, B. Rapid mapping based on UAV digital aerial photography system. Research, 2016(9):20-20.

[19] Zhao, D.W.; Tang, B.L. Zhang, C. Discussion on application of aerial photography based on UAV. Natural Science: Full Text 2016(9):194-194.

[20] Zhang, L.; Li, B.B. Application of STK in UAV route planning. Automation and Instrumentation 2016(11):151-153.

[21] Qiu, G.Q.; Yang, Z.L.; Yang, L.; et al. A network node design of wireless sensor based on ZigBee protocol. Automation and Instrumentation 2008(3):10-11+66.

[22] Yu, Z.G.; Wan S.Q.; Chen, Z.F.; et al. Design of low power and multi-functional data acquisition system based on wireless sensor network. Journal of China Academy of Electronics and Information Technology 2012, 7(2):124-128.

[23] Wang, T.; Liu, S.; Ge, L.S. Design of High-speed wireless communication receiver in parallel DC/DC system journal of power supply 2018, 16(1):166-170.

[24] Bi, K.; Li, Y.C.; Ding, X.B.; et al. Aerial photogrammetric technology of light 
small UAV: status and trend of development. Bulletin of Surveying and Mapping 2015(3):27-48.

[25] Liu, P.; Peng, Y.P.; Zou, X.Q.; et al. Present situation and prospect of unmanned aerial photogrammetric system in china. Geospatial Information 2010, 08(4):4-6.

[26] Banerjee, A. and Bej, S. On Extension of Regular Graphs. Journal of Discrete Mathematical Sciences and Cryptography, 2018; 21(1):13-21.

[27] Ellison, M., Calinescu, R. and Paige, R.F. Evaluating Cloud Database Migration Options Using Workload Models. Journal of Cloud Computing, 2018; 7(1).

[28 Promsakon, C. Edge Colorability of Unitary Endo-Cayley Graphs of Cyclic Groups. Journal of Discrete Mathematical Sciences and Cryptography, 2018; 21(1):191-198.

[29] Narasimman, P. Solution and Stability of a Generalized Kadditive Functional Equation. Journal of Interdisciplinary Mathematics, 2018; 21(1):171-184.

[30] Buch, V.R., Rawal, S.K. and Chawla, A. Effect of Sputtering Pressure On Structural, Optical and Electrochromic Properties of Tungsten Oxide Film Deposited by SputterinNE.Refg. Journal of Mechanical Engineering Research and Developments, 2018; 41(1):78-85.

[31] Saraf Esmaili, S., Maghooli, K. and Nasrabadi, A.M. A New Model for Face Detection in Cluttered Backgrounds Using Saliency Map and C2 Texture Features. International Journal of Computers and Applications, 2018; 40(4):214-222. 